TP Periodica Polytechnica

Mechanical Engineering

59(3), pp. 143-152, 2015

DOI: 10.3311/PPme. 7888

Creative Commons Attribution (i)

RESEARCH ARTICLE

\section{DES Configurators for Rapid Virtual Prototyping and Optimisation of Manufacturing Systems}

\author{
Csaba Haraszkó ${ }^{1 *}$, István Németh ${ }^{1}$
}

Received 06 January 2015; accepted after revision 03 June 2015

\begin{abstract}
In the framework of the COPERNICO research project configurators were developed under the Siemens Tecnomatix Plant Simulation software environment, which support the rapid creation of the discrete event simulation (DES) models of several types of manufacturing systems. With the use of the configurators after defining the required input data - such as the product mix, production processes, layout prototype, etc. - the DES models of the manufacturing system alternatives will be automatically created. The layout prototypes are based on a cladistics classification of manufacturing systems. The aim of the configurators is not only to purely create the simulation models but also to create models that can be used for further optimisations, that can be done e.g. by using the built in genetic algorithms tool, based on specific objectives. In this paper some research results are demonstrated on how the configurators create DES models and how the models can be applied.
\end{abstract}

\section{Keywords}

DES configurators, manufacturing systems, cladistic classification, discrete event simulation, product sequence optimisation

\footnotetext{
${ }^{1}$ Department of Manufacturing Science and Engineering, Faculty of Mechanical Engineering,

Budapest University of Technology and Economics,

H-1111 Budapest, Müegyetem rkp. 3., Hungary

*Corresponding author, e-mail: haraszko@manuf.bme.hu
}

\section{Introduction}

Today the design or re-design process of manufacturing systems requires wider and deeper integration of design methodologies and software tools. The design process should include the synthesis, analysis and optimisation tasks integrating the equipment selection and the layout planning activities as well. In order to achieve that integrated models should be developed that represent the products, processes and manufacturing equipment in a virtual environment. Several parameters - such as the product mix, control strategies of material flow, layout types, parameterised existence of equipment etc. - should be represented in these integrated models in order to be able to optimise the key performance indicators in a more comprehensive way. Small companies cannot afford investing in expensive modelling systems and employing personnel to design their new manufacturing facilities or model and evaluate their existing manufacturing systems. Therefore, affordable, easy-to-use modelling tools are needed that support the rapid design of manufacturing systems. The COPERNICO research project [1] aimed to achieve these goals. The major technical objectives of the project were to develop a cladistics based classification system of manufacturing systems, and to develop several software tools to support the rapid design, simulation, visualisation and evaluation of manufacturing systems. In the framework of the project configurator software tools were developed that support the rapid creation of the discrete event simulation (DES) models of several types of manufacturing systems.

This paper first presents a literature survey of using DES for the design of manufacturing systems. Then the layout prototypes that serve the basis for systematic layout design are described. These layout prototypes were defined using the species derived by the COPERNICO cladistic classification system. And at last the paper describes the main functions and the internal structure of the DES configurators that support the rapid creation and evaluation of the DES models of manufacturing systems, and it shows an example of performing optimisation with automatically created DES model. 


\section{The use of Discrete Event Simulation in design of Manufacturing Systems}

Simulation has been widely used to support decisions in planning, implementing, and operating of manufacturing systems. The general topic of simulation and the use of different simulation software tools have been addressed in several textbooks (e.g. [2-4]).

Most commercial simulation software tools for ComputerAided Production Engineering are discrete event simulators. A survey on the use of DES in manufacturing systems has been presented in [5], where the applications of simulation were classified into two categories: manufacturing system design and manufacturing system operation. In the category of manufacturing system design, simulation is used to evaluate long term decisions. This category is subdivided into facility design, material handling system design, manufacturing cell design, and flexible manufacturing system design. The second category focuses on operations planning, scheduling, real-time control, operating policies, and performance analysis. The survey concluded that with the increase of the computational power of computers, the time required to construct and run simulations decreased and hence, simulation started to be used more often in operational applications. Kelton [6] arrived at a similar conclusion by arguing that simulation moved from a design tool to managerial decision support tool with the development of software applications.

Many publications have discussed the role of simulation in digital manufacturing (e.g. [7]) and different aspects of simulation-based design of manufacturing systems (e.g. [8, 9]).

There exist several commercial DES software tools. The most known ones are Anylogic by Anylogic [10], Arena by Rockwell Automation [11], FlexSim by FlexSim Software Products [12], Plant simulation by Siemens [13] and Witness by Lanner [14]. These software tools have already been evaluated (e.g. $[15,16])$ based on various aspects which helps the user to choose the right one for certain simulation task.

The current DES systems are mainly used to support the analysis and optimisation phases of manufacturing systems design. It means that DES models are built to simulate certain behaviours of an existing or a future manufacturing system. The current DES software tools are lack of the ability to support the systematic design of manufacturing system. It means, that the current DES tools should be extended with the capability of supporting the synthesis phase of the design process that will increase the divergence in the design process through generating several design alternatives, thus providing the search and optimisation methods with a wider search space. This requires some automation in the model creation phase.

The automated generation of simulation models has already been in focus of some research works. Huber et al. [17] developed algorithms and a tool ( $\left.\mathrm{d}^{3} \mathrm{FACT}\right)$ to automatically generate simulation models by using stochastic techniques. The size, structure, layout and behaviour of the generated model can be parameterised. Their tool can build models hierarchically; parameterise components and link them to each other according to the input parameters. With this tool the usability of the created model may be limited if the user wants to modify it in big extent. Aggogeri et al. [18] described the manufacturing systems as a group of processes governed by the engineering strategies and technologies. The idea is to realise a model that is able to be automatically adapted to the user's specific needs. The model must be characterised by a high level of flexibility, configurability and adaptability in order to automatically simulate multiple/ heterogeneous industrial scenarios. An adaptable and flexible DES model has been developed to represent different manufacturing environments. The goal of their proposed DES-based parametric model is to offer to industrial analysts a compact, fast and easy way to configure and use decision support tools.

The DES Configurators presented in the paper can rapidly create rudimentary DES models composed of several layout arrangement variants and control strategies by using some predefined layout templates. These generated models can be refined or evolved by modifying the attributes of the components or building up a more complex system model from the automatically created subsystems using the standard modelling support of the commercial DES software tools, such as programmable software environment or graphical user interface. The main advantage of this approach is that small as well as huge models can be easily generated with all the necessary elements by using the configurators instead of the traditional drag $\&$ drop and manual programming way.

\section{Layout prototypes of manufacturing systems}

Within the COPERNICO project a cladistics based classification of manufacturing systems was developed. Cladistics is an evolutionary classification scheme that not only describes the attributes of existing entities but also the ancestral characteristics. Cladistic classification of manufacturing systems distinguishes present from past systems and may assist in validating emerging new systems. The application of cladistics to manufacturing systems has opened up a new way to classify and link evolutionary changes to main manufacturing changes of the past and present. A comprehensively constructed cladogram provides a blueprint that can be used as a guide to help changing present structures. Using the cladistic approach, the evolutionary relationships between fifty-three candidate species of manufacturing systems, using 'descriptors' drawn from a library of thirteen characters with a total of eighty-three states, were hypothesized, described and presented diagrammatically. The manufacturing species were then organised in a hierarchical classification with fifteen genera, six families and three orders under one 'class' of discrete manufacturing [19, 20].

Presenting the cladistics classification system is out of the scope of this paper. As an example Fig. 1 shows a portion of the hierarchical classification. In the framework of the 


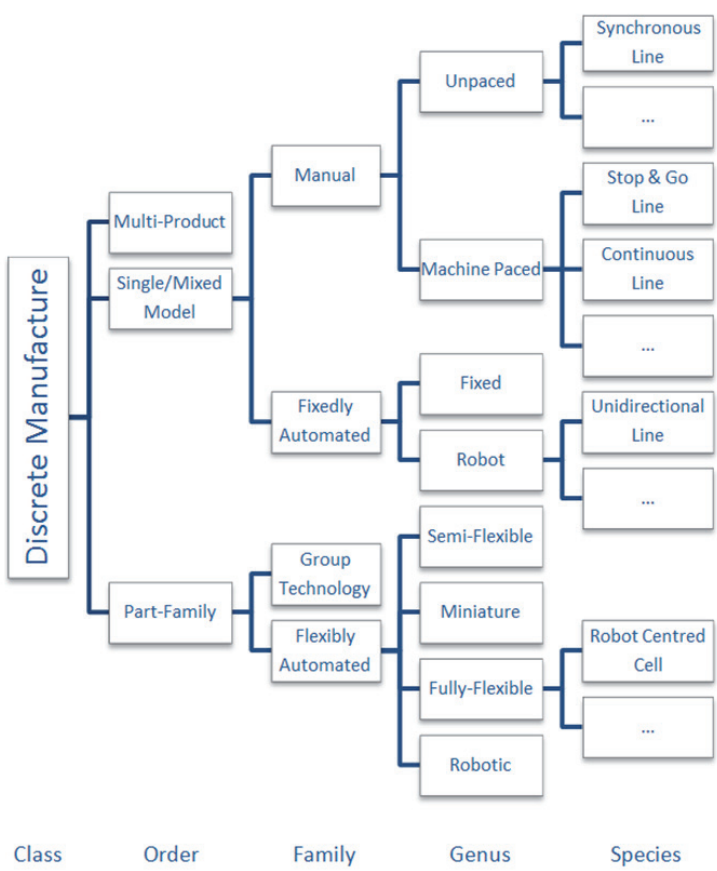

Fig. 1 Part of a Hierarchical Classification of Discrete Manufacturing Systems

COPERNICO project DES configurators of four species have been developed; they are shown in the right column of Fig. 1. In this paper the configurator developed for the species so-called 'Machine Paced Stop \& Go Line' is presented in more detail. It has evolved from a common ancestor and is sharing many character states of the species 'Unpaced Synchronous Line'. But these two species are differentiated by an automated primary material handling system (PMHS). Specifically, the Machine Paced Stop \& Go Line has an intermittent PMHS where the conveyor, in-line cart, etc., stops for every process/workstation. Specimens are typically found when the product is of moderate size and includes, for example, over 30 workstations; processing time at each workstation is between say 30 seconds and several minutes (Takt timed) [19].

Some of the layout species were selected as layout prototypes for rapid systematic layout design. Then further layout sub-species (or sub-types) of each of the selected species were identified by the authors. Based on the typical characters and states of the certain layout type (species), algorithms and DES configurator software tools were developed that can generate simulation models based on these layout templates.

\section{The DES Configurators}

The aim of the DES configurators is to automatically create the DES models of the manufacturing systems. It means that the manufacturing processes such as type and order of operations can be described, the type of manufacturing equipment, the type of material flow solutions and the material handling systems, and the layout type (species and sub-species or sub-types) can be selected, and the product mix can be defined. Then the arrangement of the manufacturing system components and all the objects necessary to simulate the manufacturing processes can be automatically created. The DES configurators have been developed under the Siemens Tecnomatix Plant Simulation software package [21]. The built-in programming language SimTalk of Plant Simulation has been used for software development. The main functions of the configurators are described in the remaining part of this section. Then in Section 5 the configurator made for the Stop\&Go species will be presented in more detail.

A part of the internal structure of the tool library within Plant Simulation can be seen in Fig. 2. The LD (Layout Design) directory contains the main elements, dialogs and methods for creating the DES model.

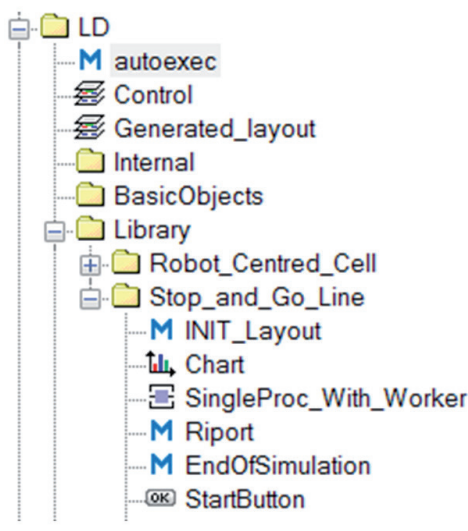

Fig. 2 A part of the internal structure

On the first tab of the graphical user interface (GUI) the products to be produced can be defined (shown in Fig. 3). After pressing the button 'PRODUCT INFO TABLE' the names, their sequence and the amount of each product can be defined on a dialog matrix. This initial product sequence may be modified later on during the application of the generated DES model.

On the second tab of the GUI the production stations can be configured (shown in Fig. 4). The stations/equipment can be selected from a database (using a drop down list) or directly typed in (e.g. milling, drilling, turning, etc.), then the process time table and setup time matrix for all products on each station can be specified.

The order of stations for each product can also be defined, but the existence of this option depends on the layout type. Based on the defined stations the components of the manufacturing system model will be automatically created and parameterised by the configurator later on. As a result we have the capability to easily generate DES models of various types and sizes; even huge models can be rapidly created using the configurator instead of the manual drag \& drop method.

On the third tab of the GUI the type of manufacturing system layout can be selected (shown in Fig. 5). On this panel it is possible to select the species of the discrete manufacturing systems identified by the cladistics classification system (see Section 3). The selection can be done by selecting the order, family, genera, and then the species. The available species can also be selected from a list directly. 


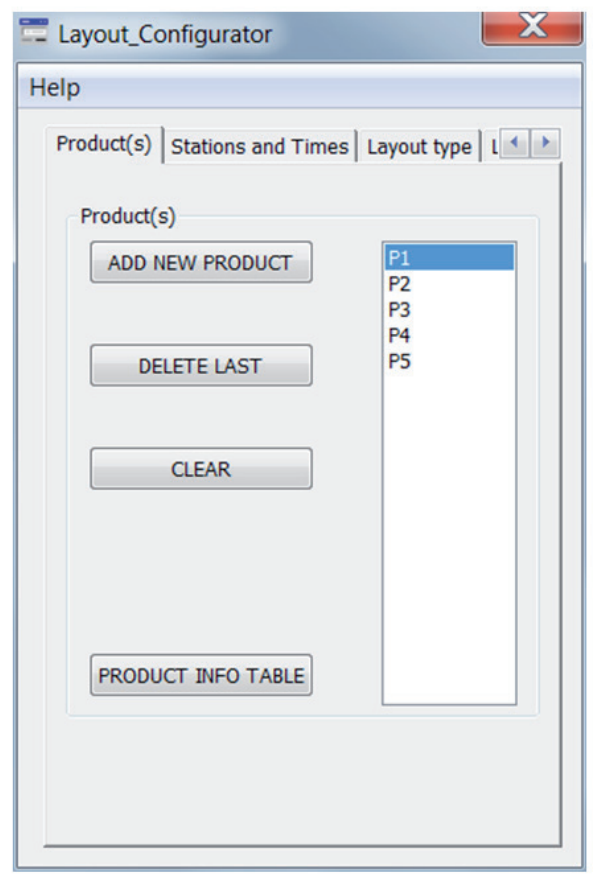

Fig. 3 DES configurator: 'Product(s)'

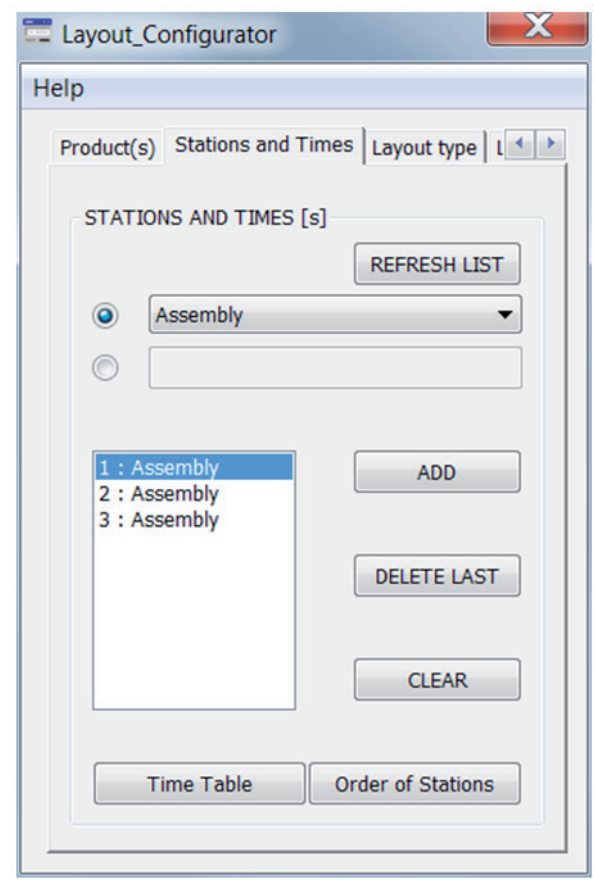

Fig. 4 DES configurator: 'Stations and Times'

By pressing the button 'ADVANCED' more detailed options can be found such as selecting the sub-species of the selected species. Configurator software tools were developed for several sub-species of the following layout species (see Fig. 1 as well): 'Manual Machine Paced Stop \& Go Line'; 'Continuous Line'; 'Unidirectional Line'; and 'Robot Centred Cell'. For example the sub-species of the species 'Robot Centred Cell' are the following:

- 'Line' (Base 1), where the stations are in line with a portal robot to serve them;

- 'Circular' (Base 2), where the stations are in a circle arrangement with a humanoid robot in the centre to serve them;

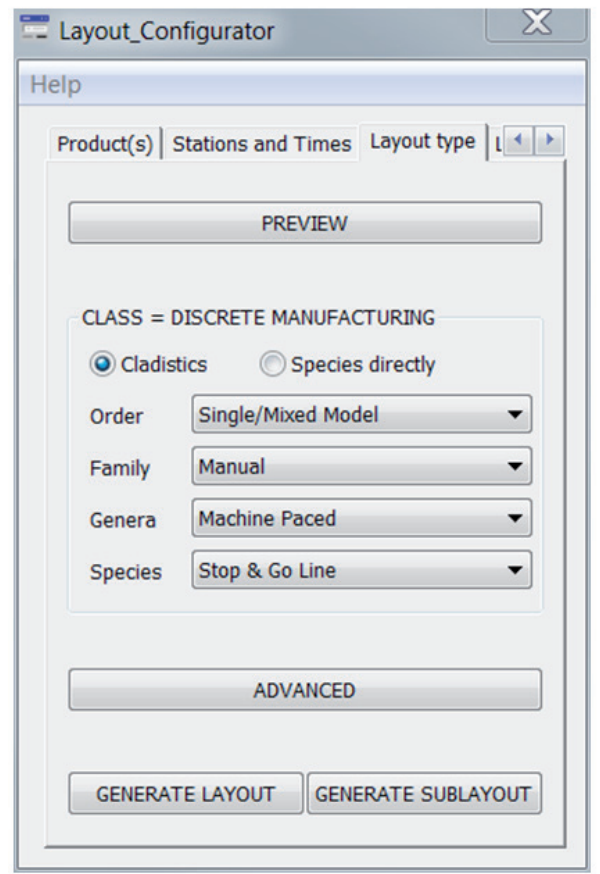

Fig. 5 DES configurator: 'Layout type'

- 'Multi-line Dependent-Base', it means e.g. from the Line layout there are more instances in parallel and they are dependent on each other by the portal robot;

- 'Multi-line Independent-Base', it means e.g. from the Circular layout there are more instances in parallel and they are independent on each other, i.e. they have own robots;

- 'Multi-line Independent-Dependent-Base', it means that from the dependent sub-type we can generate more than one in parallel and they are independent on each other.

Other layout specific data can also be defined within this dialog such as the radius and the angle of the layout in case of the Circular 'Robot Centred Cell'.

After selecting and defining the most relevant data described above, within this module the functional simulation model of the system can be created easily by pressing the button 'GENERATE LAYOUT'.

It is important to note that the simulation models created by the configurator contain not only topological information of the layout but specific control logics for the material flow as well; therefore, the created DES models can be immediately used for performing simulations or can be further modified based on several other considerations such as 3D arrangement aspects [22].

\section{The Stop\&Go DES configurator}

This section first describes the configurator developed for DES model creation of 'Manual Machine Paced Stop \& Go Line' layout types in detail, then it presents an example of the application of the generated models for optimisation.

The typical characteristics of the manufacturing systems of the Stop\&Go line based on the $1^{\text {st }}$ Generation Factual Cladistic Classification of Discrete Manufacturing Systems [19] are as follows: 
- Multi-product/multi order capability;

- Entrepreneurial management;

- Operator performs significant specialised processes, produces part of product;

- Buffer (work-in-progress) between processes;

- Extensive universal process capability (i.e., primarily hand/ power tools, mechanised/CNC machine tools/centres);

- Operator performs single or very limited set of processes;

- Centralised with routine production resource scheduling;

- Single/Mixed product model;

- Product layout;

- Line balanced minimising in-progress buffers (work-inprogress);

- Automated primary material handling system (PMHS), which stops for every process/workstation.

The typical optimisation task of this type of manufacturing systems is to determine the product sequences on both lines so that the workers' travelling distances between the stations is minimal. The aim of the Stop\&Go DES configurator is to automatically create the DES model of a user defined layout of the Stop\&Go type manufacturing system and to create and preconfigure the optimisation methods within the DES model for the product sequence optimisation problem.

\subsection{Model generation}

Figure 7 shows an example DES model created by the Stop $\&$ Go configurator. The main functions of the Stop\&Go DES configurator are the following.

The sequence and the amount of the products to be produced must be defined by using the configurator. These data are stored in a table on the Control frame of the configurator. After that the Stations and the set up and process time values of each product on every station have to be defined as well, these data are stored similarly in the Control frame. After selecting the layout species by pressing the button 'ADVANCED' further options can be specified (shown in Fig. 6).

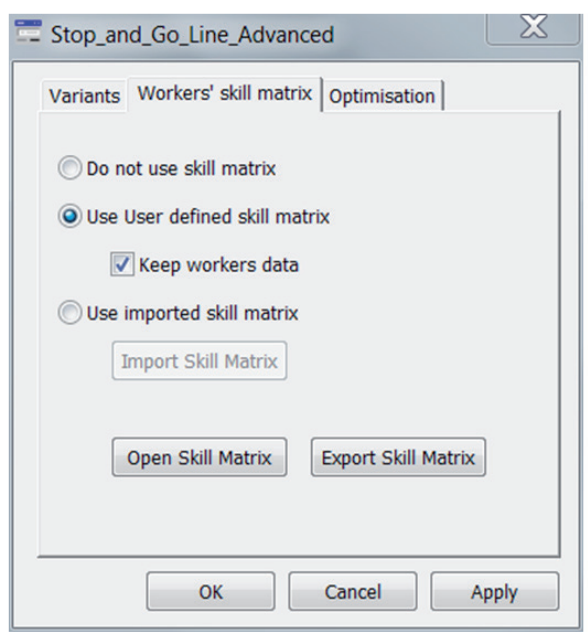

Fig. 6 DES configurator: Options of the 'Stop\&Go' layout species
The sub-species of the 'Stop\&Go' species are the following (Fig. 6, on the tab Variants):

- Line (Base), where the stations are in line with the workers;

- 'Multi-line Dependent-Base', it means that from the Line layout there are more instances in parallel and they are dependent on each other while the workers can perform activities at both lines;

- 'Multi-line Independent-Base', it means that from the Line layout there are more instances in parallel and they are independent on each other;

- 'Multi-line Independent-Dependent-Base', it means that from the dependent sub-type we can generate more than one instances in parallel and they are independent on each other.

In the example presented in Fig. 7 the selected sub-type is 'Multi-line Dependent-Base' with two lines.

Other species specific parameters can be defined such as the workers' skill matrix (Fig. 6) that defines which worker can perform activity on which stations. The first option in Fig. 6 means that we do not use special skill matrix but each station has a worker. The second option means that we can specify which worker can work on which station (see Table 1 and Table 2). And the third option means that we can import the skill matrix from other software in a specific format.

On the tab Optimisation the user can select from preconfigured objective functions such as minimize the traveling distance of the workers or minimize the throughput time.

After pressing the button 'GENERATE LAYOUT' (Fig. 5) a temporary frame of the proposed layout model will be created where the configurator creates the simulation objects based on the input data gathered so far and on the properties of the Stop\&Go layout template. The configurator creates the necessary number of objects that represents the workstations, conveyors, workplaces, workers, makes the required arrangements of the stations, and creates the control strategies, as well as charts for analysis and the GAWizard object.

For our example the following data set were specified by using the configurator: Five products to be produced defined by their name, amounts and sequence. Three assembly stations on each line are defined by process times for each product on each line and set up times. The layout species 'Stop \& Go Line' and the sub-species 'Multi-line Dependent-Base' as described above. The skills of the workers per stations were defined and the optimisation objective was selected (see the next section).

In the simulation model the assumption is that every station is connected to the adjacent one, the exact workers footpaths and the length of the conveyors were neglected, however, these abstractions can be refined later on. If it is needed these parameters and many others can be easily modified when the DES model is created (if we want to use it as a starting model) so then we can get more advantages of the great support of the standard graphical user interfaces and features of Plant Simulation. 
The main elements of the created model are:

- manufacturing system elements: conveyor (Line), workstations (Station), foot path (FootPath), workplace (Workplace);

- data table of products scheduling (Delivery), definitions of the products (P1-P5);

- data tables of the setup times and process times (SetupMatrix and ProcTime);

- input and output (Source and Drain), event controller (EventController);

- workers (WorkerPool), broker (Broker);

- other elements are created for realising the control strategies, generating report at the end of the simulation (Methods: Init, EndOfSimulation and Riport; some variables), a chart element for analysing the utilisation of the stations (ChartOfStations);

- preconfigured genetic algorithm object (GAWizard; see below).

The simulation model operates as follows. The workpieces are generated in the order of the defined sequences and amount at the sources. The conveyors transport the products from the left to the right. When a product reaches a station, then the broker (which has own built in control logic) sends a competent worker (who is able to work on that station and who is available) to the corresponding station. The worker will sets up the station and works on it, the process time values are also defined previously. If the worker has finished a station and there are no other workpieces waiting to enter the station, the worker will be released. If another station requires that worker, the broker will send the worker there, if it does not then the worker will go back to the workerpool and wait for further instructions.

The presented example model has 5 workers (in the WorkerPool) and two of them can perform activities on two stations as it is shown in Table 1 and Table 2, which means that the workers skills is assigned to the stations (e.g. Station3_2 means the third station on the second line). When defining ' 1 ' in the table means that the worker (each line is a worker in the tables) can work on that station. In the generated Plant Simulation model the activities on specific stations are called 'Additional Services'.

Table 1 The workers' services when defining as input

\begin{tabular}{|c|c|c|c|c|c|}
\hline & $\ldots$ & Station1_2 & Station2_2 & Station3_2 & $\ldots$ \\
\hline \multicolumn{6}{|l|}{1} \\
\hline 2 & & & & 1 & \\
\hline \multicolumn{6}{|l|}{3} \\
\hline 4 & & 1 & & 1 & \\
\hline 5 & & & 1 & & \\
\hline
\end{tabular}

Table 2 The workers' services in the generated model

\begin{tabular}{llc}
\hline \multicolumn{1}{c}{ Additional services } \\
\hline 1 & Station1_1 \\
2 & Station2_1 & Station3_2 \\
3 & Station3_1 & \\
4 & Station1_2 & Station3_2 \\
5 & Station2_2 & \\
\hline
\end{tabular}

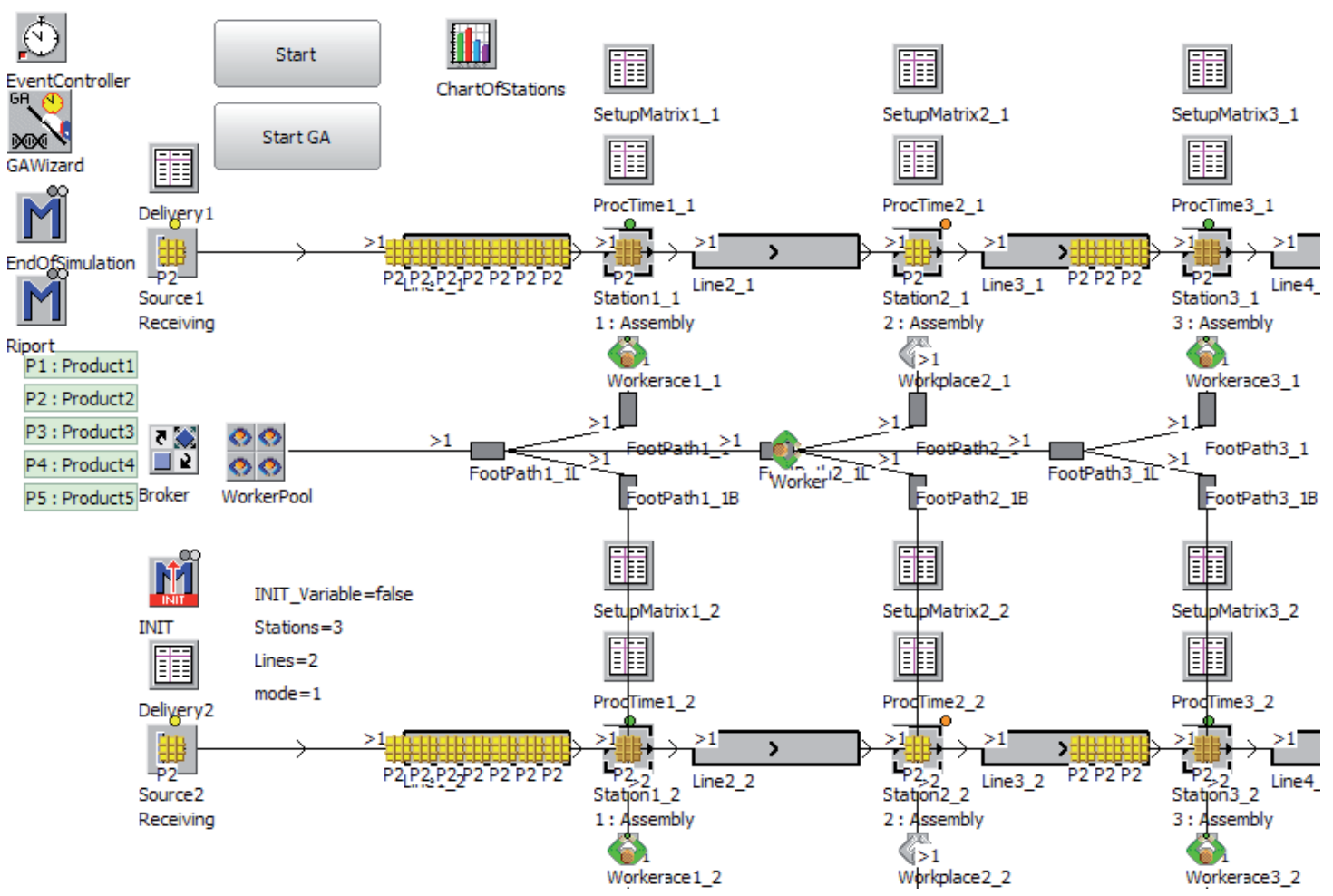

Fig. 7 Example DES model created by the configurator; layout type 'Stop \& Go Line', sub-type 'Multi-line Dependent-Base': Dependent parallel lines 
The task of the broker is to send those workers to the stations who can perform activity on them if it is preconfigured (using the special skill matrix shown in Table 1 and Table 2). A worker sets up a station and works on it until the product is ready to go to the next workstation. If there is no other product on the station, the worker can behave in the following manner:

- The worker goes back to the worker pool and will wait for instructions from the broker;

- The worker stays on the station until there is a product before the station on the line which has not zero process time on that station;

- The broker will send those workers to the stations who can perform activities in the best quality;

- The broker will send the nearest worker (who can work on it) to that station;

- Combination and weighting of the previous logics.

These options can be configured by the configurator, and the methods of the control logics of the material flow are created in the DES model according to the selected option.

When the created model is used and a simulation is completed the resource statistics can be analysed. An example of resource statistics of the model depicted in Fig. 7 is shown in Fig. 8.

\subsection{Optimisation}

The Stop\&Go DES configurator creates GAWizard object with which customised product sequence optimisations can be performed. The GAWizard object is a built-in Genetic Algorithm (GA) module of Plant Simulation (shown in Fig. 9). Genetic algorithms (GAs) are robust methods for search and optimisation that imitates the mechanisms discovered by genetics [23]. Literature of GAs and their application can be found in several text books such as [24], and [25]. GAWizard can be used to optimise several system parameters, such as throughput, throughput time, energy consumption, buffer capacity, workers' travelling distances, etc. For the optimisations many potential objectives and their combinations may be defined. Some of these objective functions can be preconfigured by using the DES Configurator.

Within the GAWizard the fitness function, the optimisation parameters and some control parameters can be defined (Fig. 9). The DES configurator generates a preconfigured GAWizard within the DES model (see Fig. 7), so that the user can immediately run optimisations or he/she may change some settings. In the preconfigured GAWizard the aggregated sum of the workers' travelling time - that is an attribute of the broker object (statMediationTime), which can collect the time of the workers' travel - was chosen directly as the fitness function (see Fig. 9) and the optimisation parameters were set to be the product sequences specified in the delivery tables (see Delivery1 and Delivery2 in Fig. 7) for each production line (see Fig. 9). In our example, there are five products on two production lines, so the GAWizard is preconfigured with these data.

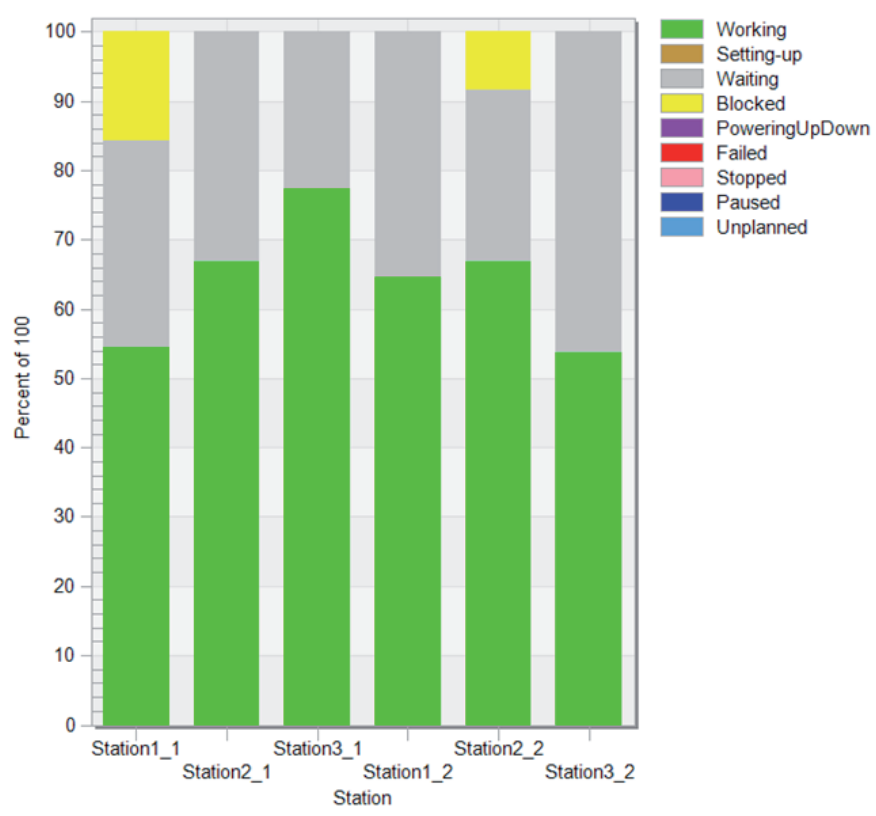

Fig. 8 Resource statistics

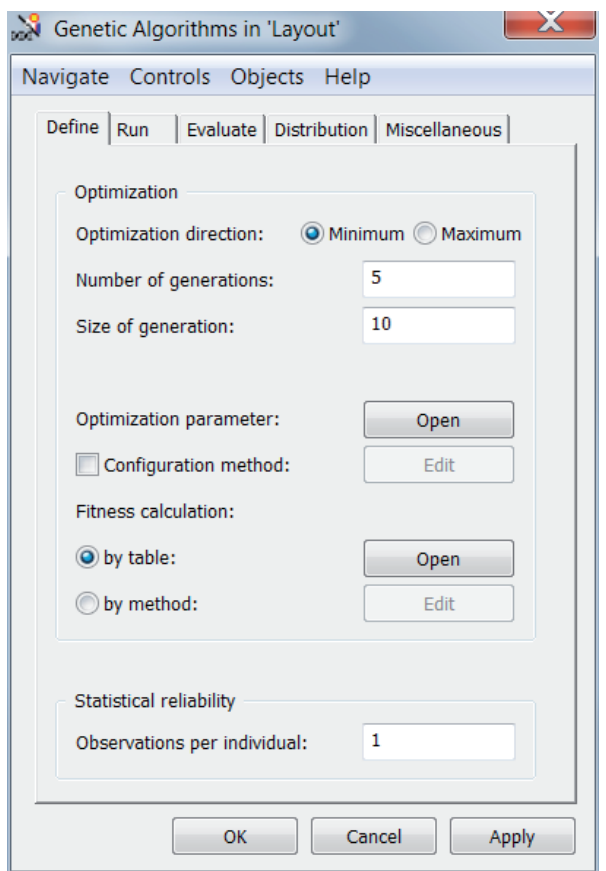

Fig. 9 Built-in Genetic Algorithm module (GAWizard) of Plant Simulation

The GAWizard was set for minimising the fitness value. The default data representation of chromosomes and the crossover and mutation operators of GAWizard were not changed.

The model with the preconfigured GAWizard can provide appropriate product sequences in each line and calculates the workers' travelling distances. The GA will search for the optimal product sequence for each line so that the workers' travelling time will be minimal. For stochastic simulation the individuals should be evaluated by several simulation runs; in this case we did not set stochastic parameters in the model; therefore, the observation per individual can be one. We can enter the 'Number of generations' and 'Size of generation' values on the tab Define as well (Fig. 9). 
Table 3 Initial product sequence for both production lines; Initial fitness value: 2:24:07.4551 (h:min:sec)

\begin{tabular}{ccc}
\hline & Number of product & Name of product \\
\hline 1 & 10 & $\mathrm{P} 1$ \\
2 & 25 & $\mathrm{P} 2$ \\
3 & 30 & $\mathrm{P} 3$ \\
4 & 15 & $\mathrm{P} 4$ \\
5 & 40 & $\mathrm{P} 5$ \\
\hline
\end{tabular}

Table 4 Optimal product sequences for Line1; Optimal fitness value: 2:05:18.0192 (h:min:sec)

\begin{tabular}{ccc}
\hline & Number of product & Name of product \\
\hline 1 & 30 & $\mathrm{P} 3$ \\
2 & 15 & $\mathrm{P} 4$ \\
3 & 25 & $\mathrm{P} 2$ \\
4 & 10 & $\mathrm{P} 1$ \\
5 & 40 & $\mathrm{P} 5$ \\
\hline
\end{tabular}

Table 5 Optimal product sequences for Line2

\begin{tabular}{ccc}
\hline & Number of product & Name of product \\
\hline 1 & 15 & $\mathrm{P} 4$ \\
2 & 25 & $\mathrm{P} 2$ \\
3 & 10 & $\mathrm{P} 1$ \\
4 & 40 & $\mathrm{P} 5$ \\
5 & 30 & $\mathrm{P} 3$ \\
\hline
\end{tabular}

Table 6 Fitness values of the first generation of the GA

\begin{tabular}{ccc}
\hline & Individual & Observations \\
\hline 1 & Gen 1 Ind 1 & $2: 24: 07.4551$ \\
2 & Gen 1 Ind 2 & $2: 48: 32.1210$ \\
3 & Gen 1 Ind 3 & $2: 13: 56.3174$ \\
4 & Gen 1 Ind 4 & $2: 18: 28.8922$ \\
5 & Gen 1 Ind 5 & $2: 10: 58.3832$ \\
6 & Gen 1 Ind 6 & $2: 42: 51.0132$ \\
7 & Gen 1 Ind 7 & $2: 25: 14.3114$ \\
8 & Gen 1 Ind 8 & $2: 15: 39.6084$ \\
9 & Gen 1 Ind 9 & $2: 44: 15.7066$ \\
10 & Gen 1 Ind 10 & $2: 47: 16.9737$ \\
\hline
\end{tabular}

Table 7 The best five results of the GA

\begin{tabular}{ccc}
\hline & Individual & Fitness \\
\hline 1 & Gen 5 Ind 4 & $2: 05: 18.0192$ \\
2 & Gen 5 Ind 14 & $2: 07: 08.7365$ \\
3 & Gen 5 Ind 12 & $2: 07: 09.3114$ \\
4 & Gen 5 Ind 7 & $2: 07: 25.8886$ \\
5 & Gen 2 Ind 8 & $2: 08: 12.4263$ \\
\hline
\end{tabular}

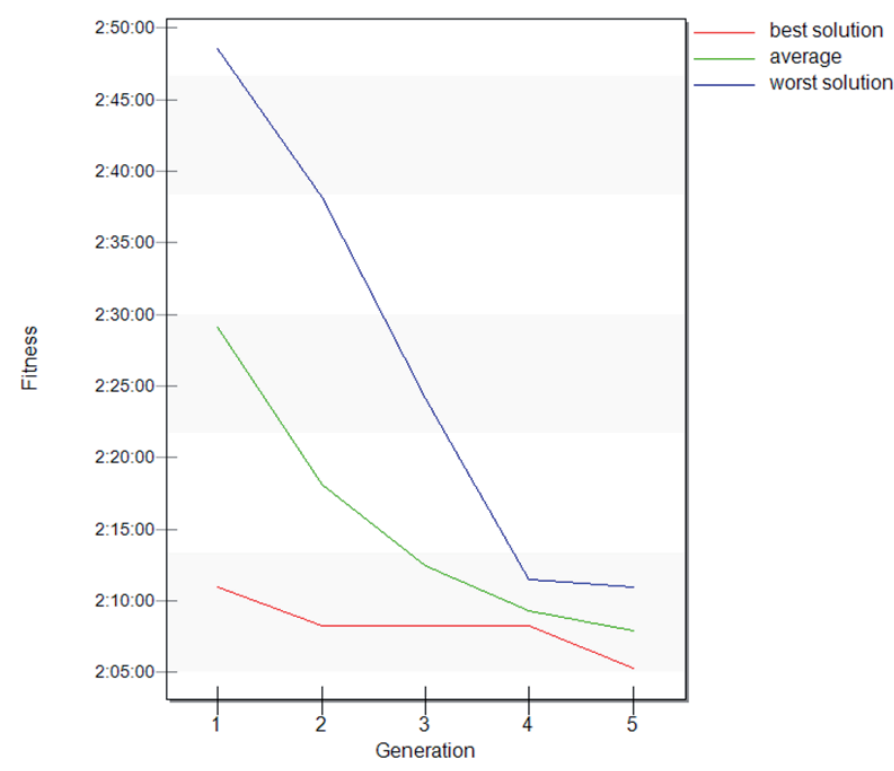

Fig. 10 Performance graph of the genetic algorithm

In the first GA generation Plant Simulation evaluates the number of generations which is entered as the 'Size of generation'. In each of the following generations it has to evaluate twice as many individuals. The number of simulation runs, which the GA module executes, results from this formula: Number of simulation runs $=$ observations per individual $*$ (generation size $+2 *$ generation size $*$ (number of generations -1)) [21]. In this case Plant Simulation has to execute 90 simulation runs $(1 *(10+2 * 10 * 4)$. The initial product sequence for both lines and the value of the fitness function for the genetic algorithm are presented in Table 3, while the optimal product sequences and the value of the fitness function are presented in Table 4 and Table 5, respectively.

It can be seen that changing the product sequences on the lines affects the travelling time (the objective function).

Table 4 and Table 5 show the optimisation results: the optimal product sequences on Line 1 (upper production line in Fig. 7) and Line 2 (lower production line in Fig. 7), respectively. Table 6, Table 7 and Fig. 10 show the evolution of the optimisation. Table 6 presents the fitness value of the individuals of the first generation.

The best five results of the optimisation are shown in Table 7 where the individuals (these are in fact the chromosomes managed by the GAWizard) represent the product sequences on both lines. The value of the optimal (minimal) workers' travelling time is 2:05:18.0192 (h:min:sec) which is the fitness value of Individual 4 of Generation 5; the best product sequences belonging to this individual are shown in Table 4 for Line 1 and Table 5 for Line2.

We note here that the optimisation took 90 simulations in this case. The brute-force search would take $5 ! * 5 !=14.400$ simulations to calculate all the possible sequence combinations.

Figure 10 depicts the performance graph of the genetic algorithm. The vertical axis of the performance graph presents 
the values of the fitness function, while the horizontal axis shows the number of generations (iterations). The red line (lower one) indicates the fitness value of the best solution in the population that represents the optimal product sequences at the end of the optimisation.

The configurator developed for the 'Stop\&Go' layout type and described in this section above was tested in an assembly plant of Electrolux containing ca. 500 assembly stations, where the human resource planning and optimisation is a difficult but important task, and affects the manufacturing operations, and, consequently, the overall factory efficiency and productivity. It is influenced by the following factors: 1. Workers presence/ absence. 2 . The product mix and sequence planning: batch sizes and batch sequences. 3. Workers skills in performing specific assembly operations. In the assembly plant there are almost 500 workers per shift to be allocated in 5 parallel assembly lines and in 100 workstations per each line. This planning is currently performed manually using some heuristic rules without simulation and optimisation. It can vary every day, causing loss of time and uncertainty on the efficiency of the workers' allocation. As a part of the COPERNICO project, based on this industrial case, the 'Stop\&Go' layout configurator had been developed and tested. A model of a much bigger layout was generated (based on real production data) and was validated. The details of this model is not presented in this paper because of confidentiality reasons, but the simple example presented above highlighted the main functionality of the DES configurator developed for the 'Stop\&Go' type manufacturing systems.

\section{Conclusion}

The paper has presented configurator software tools that support the rapid creation of the DES models of manufacturing systems. The DES configurators use some layout prototypes that are derived from the species of a cladistic classification system. The created DES models contain the layout together with the methods of the material flow control. Such tool can significantly ease and accelerate the systematic design of manufacturing systems since the rapid creation of several layout alternatives can be possible. The presented configurators are currently software prototypes but their extended successors will be very useful in industrial applications to help creating the DES models of the logistics or production systems to design their layout as well as simulate, evaluate and optimise their performance. So, the built in genetic algorithm tool of Plant Simulation was only use, but in parallel own problem specific GA methods are being developed and later they may replace the built in one.

The extensions of the current configurators are planned as follows:

- Inclusion of additional layout types (species) and looking for their sub-types (sub-species), and development of configuration methods to create simulation models for them;
- Development of methods to configure of more alternative variants of control logics and basic scheduling methods for the simulation models;

- Further development of methods and tools for the optimisation of certain parameters of the created simulation models;

- Development of more customisable GAs.

\section{Acknowledgements}

The authors acknowledge the support of the European Commission through the 7th Framework Programme under NMP2008-3.4-1 Rapid Design and Virtual Prototyping of Factories (COPERNICO; contract number 229025-2). The authors are grateful for James S. Baldwin who was the cladistics expert of the COPERNICO project, and for Alessandro Mazzon who facilitated the development and testing of the Stop\&Go DES configurator based on a real industrial problem.

\section{References}

[1] Copernico, 2010 "Cooperation Environment for Rapid Design, Prototyping and New Integration Concepts for Factory of the Future, EU FP7 integrated project." NMP2-LA-2010-229025; Duration: 01. 05. 2010 30. 04. 2014.

[2] Chryssolouris, G. "Manufacturing Systems Theory and Practice." 2nd Edition, New York: Springer Verlag. 2006.

[3] Law, A. M. "Simulation modeling and analysis." 4th Edition, New York: McGrawHill. 2006.

[4] Banks, J, Carson, J. S., Nelson, B. L., Nicol, D. M. "Discrete-event system simulation." 5th Edition Prentice Hall, Upper Saddle River, NJ. 2010.

[5] Smith, J. S. "Survey on the use of simulation for manufacturing system design and operation." Journal of manufacturing systems. 22 (2). pp. 157-171. 2003. DOI: 10.1016/S0278-6125(03)90013-6

[6] Kelton, W. D., et al. "Simulation with ARENA." McGraw-Hill Boston, MA. Vol. 3. 2002.

[7] Mourtzis, D., Papakostas, N., Mavrikios, D., Makris, S., Alexopoulos, K. "The role of simulation in digital manufacturing: applications and outlook." International Journal of Computer Integrated Manufacturing (IJCIM). 28 (1). pp. 3-24. 2015. DOI: 10.1080/0951192X.2013.800234

[8] Mourtzis, D., Doukas, M., Psarommatis, F. "Simulation-Based Design of Production Networks for Manufacturing of Personalised Products." In: IFIP WG 5.7 International Conference, APMS 2012. 397. pp. 301-309. 2013. DOI: 10.1007/978-3-642-40352-1_38

[9] Negahban, A., Smith, J. S. "Simulation for manufacturing system design and operation: Literature review and analysis." Journal of Manufacturing Systems. 33 (2). pp. 241-261. 2014. DOI: 10.1016/j.jmsy.2013.12.007,

[10] AnyLogic. The AnyLogic Company. [Online] Available from: http:// www.anylogic.com/ [Accessed: 28th March 2015]

[11] Arena. Rockwell Automation. [Online] Available from: https://www.arenasimulation.com/ [Accessed: 28th March 2015]

[12] FlexSim. FlexSim Software Projects Inc. [Online] Available from: http:// www.flexsim.com/flexsim/ [Accessed: 28th March 2015]

[13] Plant Simulation. Siemens Product Lifecycle Management Software Inc. [Online] Available from: http://www.plm.automation.siemens.com/en us/products/tecnomatix/plant_design/plant_simulation.shtml [Accessed: 28th March 2015]

[14] Witness. Lanner Ltd. [Online] Available from: http://www.lanner.com/ en/media/witness/witness13.cfm [Accessed: 28th March 2015] 
[15] Mourtzis, D., Doukas, M., Bernidaki, D. "Simulation in Manufacturing: Review and Challenges." In: 8th International Conference on Digital Enterprise Technology - DET 2014 Disruptive Innovation in Manufacturing Engineering towards the 4th Industrial Revolution. Procedia CIRP. 25. pp. 213-229. 2014. DOI: 10.1016/j.procir.2014.10.032

[16] Dias, L. M. S., Pereira, G. A. B., Vik, P., Oliveira, J. A. "Discrete Simulation Tools Ranking - A Commercial Software Packages Comparison Based on Popularity." In: Industrial Simulation Conference, Venice: Eurosis-ETI. pp. 5-11. 2011.

[17] Huber, D., Eberling, M., Laroque, C., Dangelmaier, W. "Stochastic Generation of Discrete-Event Simulation Models." In: Tenth International Conference on Computer Modeling and Simulation. pp. 241-246. 2008. DOI: 10.1109/UKSIM.2008.42

[18] Aggogeri, F., Faglia, R., Mazzola, M., Merlo, A. "Automating the Simulation of SME Processes through a Discrete Event Parametric Model." International Journal of Engineering Business Management. 7 (4). pp. 1-10. 2015. DOI: 10.5772/59958

[19] Baldwin, J. S., Rose-Anderssen, C., Ridgway, K. "Linnaean and Cladistic Classifications of Manufacturing Systems." In: Enabling Manufacturing Competitiveness and Economic Sustainability. Berlin Heidelberg: Springer-Verlag GmbH. pp. 22-34. 2012. DOI: $10.1007 / 978-3-642-23860-4 \_4$
[20] Baldwin, J. S., Allen, P. M., Winder, B., Ridgway, K. "Modelling Manufacturing Evolution: Thoughts of Sustainable Industrial Development." Journal of Cleaner Production. 13 (9). pp. 887-902. 2004.

DOI: 10.1016/j.jclepro.2004.04.009

[21] Siemens PLM Software. Tecnomatix Plant Simulation 11 TR2 (64-bit). Version 11.2.1

[22] Németh, I., Püspöki, J., Haraszkó, C., Baldwin, J. S. "Rapid layout design of manufacturing systems". Proceedings of the 14th International Conference on Modern Information Technology in the Innovation Processes of the Industrial Enterprises. Budapest. pp. 153-166. 2012.

[23] Németh, I. "A CAD Tool for the Preliminary Design of 3-Axis Machine Tools: Synthesis, Analysis and Optimisation." Leuven: Katholieke Universiteit. 2003. ISBN 90-5682-387-6

[24] Reeves, C. R., Rowe, J. E. "Genetic Algorithms: Principles and Perspectives, A Guide to GA Theory." New York, Boston, Dordrecht, London, Moscow: Kluwer Academic Publishers. 2002. ISBN: 1-4020-7240-6

[25] Chambers, L. D. (ed.) "The Practical Handbook of GENETIC ALGORITHMS Applications." 2nd Edition. Chapman \& Hall/CRC, Boca Raton London New York Washington. 2001. ISBN: 1-58488-240-9 